\title{
INTEGRAL TRANSFORMATIONS AND TAUBERIAN CONSTANTS
}

\author{
BY \\ RALPH PALMER AGNEW
}

1. Introduction. Let $\phi(x)$ be a measurable function for which $-1 / 2$ $<\phi(x) \leqq 1$ when $x \geqq 0, \phi(0)=1, \lim _{x \rightarrow 0} \phi(x)=1$, and $\lim _{x \rightarrow \infty} \phi(x)=0$. We assume that there is a positive number, which we shall always denote by $q_{0}$, such that $\phi\left(q_{0}\right)=1 / 2, \phi(x)>1 / 2$ when $0<x<q_{0}$, and $\phi(x)<1 / 2$ when $q_{0}<x$ $<\infty$. We suppose finally that

$$
\int_{0}^{x_{0}} \frac{1-\phi(x)}{x} d x<\infty
$$

and

$$
\int_{q_{0}}^{\infty} \frac{|\phi(x)|}{x} d x<\infty .
$$

It is of course true that our conditions are satisfied by each continuous decreasing function $\phi(x)$ which approaches 1 sufficiently rapidly as $x \rightarrow 0$ and which approaches 0 sufficiently rapidly as $x \rightarrow \infty$, but we do not need the heavier restrictions on $\phi(x)$. For example, our results have vivid, but perhaps in most cases unimportant, meanings when applied to such functions as that for which $\phi(x)=1$ when $0 \leqq x<1, \phi(1)=1 / 2, \phi(x)=1 / 4$ when $1<x \leqq 2$, and $\phi(x)=0$ when $x>2$. We do not assume that $\phi(x)$ is monotone, and we do not assume that $\phi(x) \geqq 0$, because we want our results to apply to such functions as the Riemann functions

$$
\phi(x)=\left|\frac{\sin x}{x}\right|^{r}
$$

and

$$
\phi(x)^{*}=\left(\frac{\sin x}{x}\right)^{r}
$$

where $r$ is a suitable positive parameter which is usually an integer in (1.31).

Let $u(x)$ be a complex valued function defined for $x \geqq 0$ and Lebesgue integrable over each finite interval $0 \leqq x \leqq T$. Let

$$
S(T)=\int_{0}^{T} u(x) d x
$$

Received by the editors October 22, 1951. 
and let

$$
F(t)=\int_{0}^{\infty} \phi(x t) u(x) d x, \quad t>0,
$$

whenever the integral on the right exists as a Cauchy integral $\lim _{h \rightarrow \infty} \int_{0}^{h} \phi(x t) u(x) d x$. In the theory of linear integral transformations, $\lim _{t \rightarrow 0} F(t)$, when it exists, provides a generalized value of $\lim _{T \rightarrow \infty} S(T)$; but we do not need enough restrictions on $\phi(x)$ to imply that all of the transformations are regular in the sense that $F(t)$ exists for each $t>0$ and $\lim _{t \rightarrow 0} F(t)$ $=\lim _{T \rightarrow \infty} S(T)$ whenever $u(x)$ is a measurable function for which $\lim _{T \rightarrow \infty} S(T)$ exists.

Some of the familiar transformations satisfying our conditions are that of Laplace for which $\phi(x)=e^{-x}$; those of Riesz for which $\phi(x)=(1-x)^{r}$ when $0 \leqq x \leqq 1$ and $\phi(x)=0$ when $x>1$ with positive order $r$; those of Stieltjes for which $\phi(x)=(1+x)^{-r}$ with positive order $r$; and that of Lambert for which $\phi(x)=x /\left(e^{x}-1\right)$ with $\phi(0)=1$.

In addition to the classic Abelian and Tauberian theorems which give information about one of $\lim _{t \rightarrow 0} F(t)$ and $\lim _{T \rightarrow \infty} S(T)$ when the other exists, it is possible to find elegant estimates of $|F(t)-S(T)|$ when neither $\lim F(t)$ nor $\lim S(T)$ is assumed to exist but $u(x)$ is assumed to satisfy the Tauberian condition $\lim \sup _{x \rightarrow \infty}|x u(x)|<\infty$. Such estimates were obtained by Rajagopal [1950] for the case $T=q_{0} / t$. Study of problems of this nature originated with Hadwiger [1944]. For a general treatment and references to some of the work on the subject, see Delange [1950].

It is the object of this paper to obtain estimates of $|F(t)-S(T)|$ for more general relations between $t$ and $T$ and especially to prove facts relating to the best constants in the estimates.

2. The function $A(q)$. For each $q>0$, let

$$
A(q)=\int_{0}^{q} \frac{1-\phi(x)}{x} d x+\int_{q}^{\infty} \frac{|\phi(x)|}{x} d x .
$$

Both integrands are nonnegative. Our hypotheses on $\phi(x)$ imply that $\lim _{q \rightarrow 0} A(q)=\infty, \lim _{q \rightarrow \infty} A(q)=\infty, A(q)$ is decreasing when $0<q<q_{0}$, and $A(q)$ is increasing when $q_{0}<q<\infty$. It follows that $A(q)$ has a unique absolute minimum when $q=q_{0}$. The minimum, which we shall always denote by $A_{0}$, is

$$
A_{0}=A\left(q_{0}\right)=\int_{0}^{q_{0}} \frac{1-\phi(x)}{x} d x+\int_{q_{0}}^{\infty} \frac{|\phi(x)|}{x} d x
$$

This minimum $A_{0}$ is positive except in the special case in which $\phi(x)=1$ almost everywhere in $0<x<q_{0}$ and $\phi(x)=0$ almost everywhere in $x>q_{0}$; in this special case $F(t)=S\left(q_{0} / t\right)$. We can write $A(q)$ in the form 


$$
\begin{aligned}
A(q) & =\lim _{h \rightarrow 0}\left[\int_{h}^{q} \frac{1}{x} d x-\int_{h}^{\infty} \frac{\phi(x)}{x} d x+\int_{h}^{\infty} \frac{|\phi(x)|+\phi(x)}{x} d x\right. \\
& =\Gamma+\log q+\int_{0}^{\infty} \frac{|\phi(x)|+\phi(x)}{x} d x
\end{aligned}
$$

where

$$
\Gamma=\lim _{h \rightarrow 0}\left[\log \frac{1}{h}-\int_{h}^{\infty} \frac{\phi(x)}{x} d x\right] .
$$

In the case of the Laplace transformation for which $\phi(x)=e^{-x}$, we have $q_{0}=\log 2$ and

$$
A_{0}=A\left(q_{0}\right)=\int_{0}^{\log 2} \frac{1-e^{-x}}{x} d x+\int_{\log 2}^{\infty} \frac{e^{-x}}{x} d x .
$$

The constant $\Gamma$ in (2.3) and (2.31) is Euler's constant $\gamma=.5772157$ and

$$
A_{0}=A\left(q_{0}\right)=\gamma+\log \log 2+2 \int_{\log 2}^{\infty} \frac{e^{-x}}{x} d x=.9680448
$$

These constants involved in the Laplace transforms of functions are, as should be expected, the same as the constants involved in Abel power series transforms of series; see Agnew [1945] and [1949].

In the case of the Riesz transformation of order $r>0$ for which, where the superscripts indicate the order $r$ and not derivatives, $\phi^{(r)}(x)=(1-x)^{r}$ when $0 \leqq x \leqq 1$ and $\phi^{(r)}(x)=0$ when $x>1$, we have $q_{0}^{(r)}=1-2^{-1 / r}$ and

$$
A_{0}^{(r)}=A\left(q_{0}^{(r)}\right)=\int_{0}^{1-2^{-1 / r}} \frac{1-(1-x)^{r}}{x} d x+\int_{1-2^{-1 / r}}^{1} \frac{(1-x)^{r}}{x} d x .
$$

When $r$ is a positive integer, evaluation of the integrals in (2.5) gives

$$
\begin{aligned}
& A_{0}^{(r)}=\sum_{k=1}^{r} \frac{1-2^{-k / r}}{k}+\sum_{k=r+1}^{\infty} \frac{2^{-k / r}}{k}, \\
& A_{0}^{(r)}=\log \frac{1}{1-2^{-1 / r}}-\sum_{k=1}^{r} \frac{2^{1-k / r}-1}{k} .
\end{aligned}
$$

Some approximate numerical values are

$$
\begin{array}{ll}
q_{0}^{(1)}=1 / 2, & A_{0}^{(1)}=\log 2=.69315 ; \\
q_{0}^{(2)}=.292893, & A_{0}^{(2)}=.81373 ; \\
q_{0}^{(3)}=.20630, & A_{0}^{(3)}=.86106 .
\end{array}
$$

For appraisal of $A_{0}^{(r)}$ for positive values of $r$, integer or not, we make a change 
of variable in (2.5), setting $x=1-e^{-y / r}$. Thus we obtain, for each $r>0$,

$$
A_{0}^{(r)}=\int_{0}^{\log 2} \frac{1-e^{-y}}{y} \frac{y / r}{e^{y / r}-1} d y+\int_{\log 2}^{\infty} \frac{e^{-y}}{y} \frac{y / r}{e^{y / r}-1} d y .
$$

From obvious properties of the function

$$
\frac{u}{e^{u}-1}=\frac{1}{1+u / 2 !+u^{2} / 3 !+\cdots}
$$

it follows that $A_{0}^{(r)}$ steadily increases as $r$ increases, that $A_{0}^{(r)}<A_{0}$ for each $r>0$, and that $\lim _{r \rightarrow \infty} A_{0}^{(r)}=A_{0}$ where $A_{0}$ is the constant in (2.4) and (2.41) which belongs to the Laplace transformation.

In the case of the Stieltjes transformation of order $r>0$ for which $\phi^{(r)}(x)$ $x(1+x)^{-r}$, we have $q_{0}^{(r)}=2^{1 / r}-1$ and

$$
\begin{aligned}
A_{0}^{(r)}=A\left(q_{0}^{(r)}\right)= & \int_{0}^{2^{1 / r-1}}\left[\frac{1}{x}-\frac{1}{x(1+x)^{r}}\right] d x \\
& +\int_{2^{1 / r-1}}^{\infty} \frac{1}{x(1+x)^{r}} d x .
\end{aligned}
$$

When $r$ is a positive integer, evaluation of the integrals in (2.6) gives

$$
A_{0}^{(r)}=\frac{2}{r} \log 2-\log \left(2^{1 / r}-1\right)-\sum_{k=1}^{r-1} \frac{2^{1-k / r}-1}{k} .
$$

Some approximate numerical values are

$$
\begin{array}{ll}
q_{0}^{(1)}=1, & A_{0}^{(1)}=2 \log 2=1.38629 ; \\
q_{0}^{(2)}=.414214, & A_{0}^{(2)}=1.16031 ; \\
q_{0}^{(3)}=.259921, & A_{0}^{(3)}=1.09202 .
\end{array}
$$

For appraisal of $A_{0}^{(r)}$ for positive values of $r$, integer or not we make a change of variable in (2.6), setting $x=e^{y / r}-1$. Thus we obtain, for each $r>0$,

$$
A_{0}^{(r)}=\int_{0}^{\log 2} \frac{1-e^{-y}}{y} \frac{y / r}{1-e^{-y / r}} d y+\int_{\log 2}^{\infty} \frac{e^{-y}}{y} \frac{y / r}{1-e^{-y / r}} d y .
$$

Good estimates of the order of magnitude of $A_{0}^{(r)}$ can be obtained by using

$$
\begin{aligned}
1+\frac{u}{2}<\frac{u}{1-e^{-u}}<1+u, & 0<u & \leqq 2, \\
u & <\frac{u}{1-e^{-u}}<1+u, & u \geqq 2 .
\end{aligned}
$$

We find that, when $0<r \leqq 2^{-1} \log 2$, 


$$
A_{0}^{(r)}>\int_{0}^{2 r} \frac{1-e^{-y}}{y} d y+\frac{1+2 \log 2-2 r-e^{-2 r}}{2 r}
$$

and, when $r \geqq 2^{-1} \log 2$,

$$
\text { (2.66) } A_{0}^{(r)}>\int_{0}^{\log 2} \frac{1-e^{-y}}{y} d y+\int_{\log 2}^{2 r} \frac{e^{-y}}{y} d y+\frac{1}{2 r}\left(\log 2+e^{-2 r}\right)
$$

and, on the other hand, when $r>0$,

$$
A_{0}^{(r)}<A_{0}+(1 / r) \log 2,
$$

the constant $A_{0}$ being precisely that in (2.4) and (2.41). It follows from (2.65) that $A_{0}^{(r)} \rightarrow \infty$ as $r \rightarrow 0$, and that $A_{0}^{(r)} \rightarrow A_{0}$ as $r \rightarrow \infty$. That the latter fact can be obtained from (2.62) without computations may be seen as follows. The integrands in (2.62) are positive and when $r$ increases to become infinite they decrease steadily to the integrands in (2.4). It follows that $A_{0}^{(r)}$ steadily decreases as $r$ increases, that $A_{0}^{(r)}>A_{0}$ for each $r>0$, and that $\lim _{r \rightarrow \infty} A_{0}^{(r)}=A_{0}$ where $A_{0}$ is the constant in (2.4) and (2.41) which belongs to the Laplace transformation.

In the case of the Lambert transformation for which $\phi(x)=x /\left(e^{x}-1\right)$, we can put $A(q)$ in the form

$$
A(q)=\int_{0}^{q}\left(\frac{1}{x}-\frac{e^{-x}}{1-e^{-x}}\right) d x+\int_{q}^{\infty} \frac{e^{-x}}{1-e^{-x}} d x
$$

and elementary integration gives

$$
A(q)=\log \frac{q}{\left(1-e^{-q}\right)^{2}} .
$$

In this case the constant $\Gamma$ of (2.3) and (2.31) is 0 . The value of $q_{0}$ of $q$ for which $\phi(q)=1 / 2$ and $A(q)$ is a minimum is that for which $e^{q}=1+2 q$. Thus $q_{0}=1.256431, e^{q_{0}}=3.512862$, and $A_{0}=A\left(q_{0}\right)=.898291$.

Before entering seriously into a discussion of relations between $F(t)$ and $S(T)$ we remark that preliminary ideas, some of which may be misleading, can be obtained very quickly from consideration of the special function $u(x)$ for which $u(x)=0$ when $0 \leqq x \leqq 1$ and $u(x)=x^{-1}$ when $x>1$. When $T>1$, $S(T)=\log T$. For each $t>0$

$$
\begin{aligned}
F(t) & =\int_{1}^{\infty} \frac{\phi(x t)}{x} d x=\int_{t}^{\infty} \frac{\phi(x)}{x} d x=\int_{t}^{1} \frac{\phi(x)}{x} d x+\int_{1}^{\infty} \frac{\phi(x)}{x} d x \\
& =-\int_{t}^{1} \frac{1-\phi(x)}{x} d x+\int_{1}^{\infty} \frac{\phi(x)}{x} d x-\log t
\end{aligned}
$$

and hence, where $\epsilon(t) \rightarrow 0$ as $t \rightarrow 0$, 
(2.81) $F(t)-S(T)=\epsilon(t)-\int_{0}^{1} \frac{1-\phi(x)}{x} d x+\int_{1}^{\infty} \frac{\phi(x)}{x} d x-\log t T$.

This gives the preliminary idea that we can make $F(t)$ and $S(T)$ nearly equal by giving the product $t T$ an appropriate constant value depending only on $\phi$. While the matter is of little importance, we note specifically that, for the special function, we shall have

$$
\lim _{\alpha \rightarrow \infty}[F(t)-S(T)]=0
$$

whenever $t(\alpha)$ and $T(\alpha)$ are positive functions of a parameter $\alpha$ such that $t(\alpha) \rightarrow 0$ and $T(\alpha) \rightarrow \infty$ as $\alpha \rightarrow \infty$ and

$$
t T=\exp \left\{-\int_{0}^{1} \frac{1-\phi(x)}{x} d x+\int_{1}^{\infty} \frac{\phi(x)}{x} d x\right\} .
$$

It happens that, for the Lambert transformation, the quantity in braces is 0 and the condition in (2.83) becomes $t=T^{-1}$.

3. Comparison of $F(t)$ and $S(T)$. For the case $t T=q_{0}$ the following theorem reduces to a theorem of Rajagopal [1950], except that the hypotheses of Rajagopal are considerably more restrictive than ours.

TheOREM 3.1. If $t=t(\alpha)$ and $T=T(\alpha)$ are positive functions of a positive parameter $\alpha$ such that, as $\alpha \rightarrow \infty, \lim t(\alpha)=0$ and $\lim T(\alpha)=\infty$, and if

$$
0<q_{1}=\liminf _{\alpha \rightarrow \infty} t T \leqq \limsup _{\alpha \rightarrow \infty} t T=q_{2}<\infty,
$$

then, when $\lim \sup |x u(x)|<\infty$,

$$
\limsup _{\alpha \rightarrow \infty}|F(t)-S(T)| \leqq A(q) \limsup _{x \rightarrow \infty}|x u(x)|
$$

where $q=q_{2}$ if $A\left(q_{1}\right) \leqq A\left(q_{2}\right)$ and $q=q_{1}$ otherwise. Moreover $A(q)$ is the best (that is, least) constant in the following sense. There is a real bounded measurable function $u(x)$ for which $0<\lim$ sup $|x u(x)|<\infty$ and equality holds in (3.12).

Supposing as always that $u(x)$ is integrable over each finite interval, let $\lim \sup |x u(x)|=L$ and let

$$
v(x)=x u(x),
$$

$x>0$.

The function $\phi(x t) u(x)$ is integrable over each finite interval $0 \leqq x \leqq x_{0}$ because $\phi(x t)$ is bounded and measurable and $u(x)$ is integrable. For large $x_{0}$ we have $|u(x)|<(L+1) / x$; hence $|\phi(x t) u(x)| \leqq(L+1) \phi(x t) / x$ for $x>x_{0}$ and therefore (1.2) implies that $|\phi(x t) u(x)|$ is integrable over $x_{0}<x<\infty$. Thus $F(t)$ exists for each $\alpha>0$. Since $-1 / 2 \leqq \phi(x, t) \leqq 1$ and $\phi(x t) \rightarrow 1$ as $\alpha \rightarrow \infty$ it follows from a criterion of Lebesgue that $\epsilon(\alpha) \rightarrow 0$ as $\alpha \rightarrow \infty$ when 


$$
\epsilon(\alpha)=-\int_{0}^{1}[1-\phi(x t)] u(x) d x .
$$

Assuming $\alpha$ large enough to make $T>1$, we obtain

$$
\begin{aligned}
F(t)-S(T) & =\epsilon(\alpha)-\int_{1}^{T}[1-\phi(x t)] u(x) d x+\int_{T}^{\infty} \phi(x t) u(x) d x \\
& =\epsilon(\alpha)-\int_{1}^{T} \frac{1-\phi(x t)}{x} v(x) d x+\int_{T}^{\infty} \frac{\phi(x t)}{x} v(x) d x
\end{aligned}
$$

\section{Letting}

$$
G(\alpha)=F(t)-S(T)-\epsilon(\alpha),
$$

we put (3.3) in the form

$$
G(\alpha)=\int_{1}^{\infty} K(\alpha, x) v(x) d x
$$

where

$$
\begin{aligned}
K(\alpha, x) & =-[1-\phi(x t)] / x & (1 \leqq x \leqq T) \\
& =\phi(x t) / x & (x>T)
\end{aligned}
$$

We find that

$$
\begin{aligned}
\int_{1}^{\infty} K(\alpha, x) d x & =-\int_{1}^{T} \frac{1-\phi(x t)}{x} d x+\int_{T}^{\infty} \frac{\phi(x t)}{x} d x \\
& =-\log T+\int_{t}^{\infty} \frac{\phi(y)}{y} d y
\end{aligned}
$$

and observe that as $\alpha \rightarrow \infty$ this has a limit only for special pairs of functions $t(\alpha)$ and $T(\alpha)$. Thus the theory of regular kernel transformations, as given for example by Hardy [1949, Chapter 3], does not apply to the transformation (3.32). However

$$
\begin{array}{rlrl}
|K(\alpha, x)| \leqq 1, & \alpha>0, & x \geqq 1 \\
\lim _{\alpha \rightarrow \infty} K(x, \alpha)=0, & x \geqq 1
\end{array}
$$

Moreover

$$
\begin{aligned}
\int_{1}^{\infty}|K(\alpha, x)| d x & =\int_{1}^{T} \frac{1-\phi(x t)}{x} d x+\int_{T}^{\infty} \frac{|\phi(x t)|}{x} d x \\
& =\int_{t}^{t T} \frac{1-\phi(x)}{x} d x+\int_{t T}^{\infty} \frac{|\phi(x)|}{x} d x
\end{aligned}
$$


and it follows from $\$ 2$ and the hypotheses of Theorem 3.1 that

$$
\limsup _{\alpha \rightarrow \infty} \int_{1}^{\infty}|K(\alpha, x)| d x=A(q) .
$$

The conclusions of Theorem 3.1 now become an immediate consequence of Lemma 4.1 since, by a criterion of Lebesgue, (3.51) and (3.52) imply (4.12) below.

4. A lemma on kernel transformations. The following lemma is a modification of a lemma proved by Agnew [1949] for transformations of sequences.

LEMma 4.1. Let $K(\alpha, x)$ be a real or complex valued function, defined for $\alpha>0$ and $x>1$, such that $K(x, \alpha)$ is a bounded measurable function of $x$ for each $\alpha$,

$$
\begin{array}{ll}
\int_{1}^{\infty}|K(\alpha, x)| d x<\infty, & \alpha>0, \\
\lim _{\alpha \rightarrow \infty} \int_{a}^{b} K(\alpha, x) v(x) d x=0 &
\end{array}
$$

whenever $1 \leqq a<b<\infty$ and $v(x)$ is integrable over $a \leqq x \leqq b$, and

$$
\limsup _{\alpha \rightarrow \infty} \int_{1}^{\infty}|K(\alpha, x)| d x=M .
$$

Then each real or complex valued function $v(x)$ which is integrable over each finite interval $1 \leqq x \leqq x_{0}$ and for which lim $\sup |v(x)|<\infty$ has a transform

$$
G(\alpha)=\int_{1}^{\infty} K(\alpha, x) v(x) d x
$$

such that

$$
\limsup _{\alpha \rightarrow \infty}|G(\alpha)| \leqq M \limsup _{x \rightarrow \infty}|v(x)| .
$$

Moreover $M$ is the best constant in the following sense. There is a bounded measurable function $v(x)$, which is real if $K(\alpha, x)$ is real, such that $0<\lim \sup |v(x)|$ $<\infty$ and equality holds in (4.15).

Proof of the first part of the lemma is quite trivial. Let lim sup $|v(x)|$ $=L<\infty$, let $\epsilon>0$, and choose $x_{0}>1$ such that $|v(x)|<L+\epsilon$ when $x \geqq x_{0}$. Then

$$
|G(\alpha)| \leqq\left|\int_{1}^{x_{0}} K(\alpha, x) v(x) d x\right|+\int_{x_{0}}^{\infty}|K(\alpha, x)|(L+\epsilon) d x
$$

so $\lim \sup |G(\alpha)| \leqq M(L+\epsilon)$ and (4.15) follows. To prove the second part, choose $\alpha_{1}$ and then $x_{1}$ such that 


$$
\int_{1}^{\infty}\left|K\left(\alpha_{1}, x\right)\right| d x>M-2^{-1}, \quad \int_{x_{1}}^{\infty}\left|K\left(\alpha_{1}, x\right) d x\right|<2^{-1} .
$$

When $\alpha_{p-1}$ and $x_{p-1}$ have been chosen, choose $\alpha_{p}$ such that $\alpha_{p}>\alpha_{p-1}$,

$$
\int_{1}^{x_{p-1}}\left|K\left(\alpha_{p}, x\right)\right| d x<2^{-p}, \quad \int_{1}^{\infty}\left|K\left(\alpha_{p}, x\right)\right| d x>M-2^{-p}
$$

and then choose $x_{p}$ such that $x_{p}>x_{p-1}$ and

$$
\int_{x_{p}}^{\infty}\left|K\left(\alpha_{p}, x\right)\right| d x<2^{-p}
$$

For each $p=1,2,3, \cdots$, let

$$
v(x)=\operatorname{sgn} K\left(\alpha_{p}, x\right), \quad x_{p-1} \leqq x<x_{p},
$$

where, as usual, $\operatorname{sgn} z=0$ when $z=0$ and $\operatorname{sgn} z=|z| / z$ when $z \neq 0$ so that in every case $z \operatorname{sgn} z=|z|$. Then $|v(x)| \leqq 1$ and $\lim \sup |v(x)|=1$. The transform $G(\alpha)$ of this function $v(x)$ is such that

$$
\begin{aligned}
\left|G\left(\alpha_{p}\right)\right|= & \left|\int_{0}^{\infty} K\left(\alpha_{p}, x\right) v(x) d x\right| \\
\geqq & -\int_{0}^{x_{p-1}}\left|K\left(\alpha_{p}, x\right)\right| d x+\int_{x_{p-1}}^{x_{p}}\left|K\left(\alpha_{p}, x\right)\right| d x \\
& -\int_{x_{p}}^{\infty}\left|K\left(\alpha_{p}, x\right)\right| d x \\
\geqq & -2^{-p}+\left(M-3 \cdot 2^{-p}\right)-2^{-p}=M-5 \cdot 2^{-p}
\end{aligned}
$$

It follows that

$$
\limsup _{\alpha \rightarrow \infty}|G(\alpha)| \geqq M=\limsup _{x \rightarrow \infty}|v(x)| .
$$

Since the first part of the lemma implies (4.15), we must have equality in (4.15). This completes the proof of Lemma 4.1 and hence also Theorem 3.1.

5. Further comparison of $F(t)$ and $S(T)$. The following theorem supplements Theorem 3.1.

THEOREM 5.1. If $t=t(\alpha)$ and $T=T(\alpha)$ are positive functions of a positive parameter $\alpha$ such that, as $\alpha \rightarrow \infty, \lim t(\alpha)=0$ and $T(\alpha) \rightarrow \infty$, and if one or both of

$$
\lim _{\alpha \rightarrow \infty} \inf t T=0 \text {, }
$$

$$
\limsup _{\alpha \rightarrow \infty} t T=\infty
$$

holds, then there is a real bounded measurable function $u(x)$ for which $\lim \sup |x u(x)|=0$ and $\lim \sup _{\alpha \rightarrow \infty}|F(t)-S(T)|=\infty$. 
Supposing first that (5.11) holds, we proceed precisely as in the proof of Theorem 3.1 until (3.6) has been obtained and then conclude that for large values of $\alpha$

$$
\int_{1}^{\infty}|K(\alpha, x)| d x>\int_{t T}^{\infty} \frac{|\phi(x)|}{x} d x
$$

Therefore (5.32) below holds. A similar argument shows that if (5.12) holds, then (5.32) holds. Therefore Theorem 5.1 is an immediate consequence of the following lemma of Agnew [1939, p. 711] which, like Lemma 4.1, is more general than we need for the purposes of this paper.

Lемма 5.3. Let $K(\alpha, x)$ be a real or complex valued function, defined for $\alpha>0$ and $x>1$, such that $K(x, \alpha)$ is a measurable function of $x$ for each $\alpha$,

$$
\int_{1}^{\infty}|K(\alpha, x)| d x<\infty, \quad \alpha>0,
$$

and

$$
\underset{\alpha \rightarrow \infty}{\limsup } \int_{1}^{\infty}|K(\alpha, x)| d x=\infty \text {. }
$$

Then there is a real bounded measurable function $v(x)$ such that

$$
\lim _{x \rightarrow \infty} v(x)=0
$$

and

$$
\underset{\alpha \rightarrow \infty}{\lim \sup }\left|\int_{1}^{\infty} K(\alpha, x) v(x) d x\right|=\infty .
$$

6. Tauberian constants. We now prove the following theorem in which, as always, $q_{0}$ is the value of $x$ for which $\phi(x)=1 / 2$ and $A_{0}=A\left(q_{0}\right)$ is the minimum value of $A(q)$.

Theorem 6.1. The constant

$$
A_{0}=A\left(q_{0}\right)=\int_{0}^{q_{0}} \frac{1-\phi(x)}{x} d x+\int_{q_{0}}^{\infty} \frac{|\phi(x)|}{x} d x
$$

is the least constant $A$ with the following property. If $u(x)$ is integrable over each finite interval and if lim sup $|x u(x)|<\infty$, then it is possible to choose positive functions $t(\alpha)$ and $T(\alpha)$, independent of the function $u(x)$, such that $\lim _{\alpha \rightarrow \infty} t(\alpha)$ $=0, T(\alpha)=\infty$, and

$$
\limsup _{\alpha \rightarrow \infty}|F(t)-S(T)| \leqq A \limsup _{x \rightarrow \infty}|x u(x)|
$$


This follows from Theorems 3.1 and 5.1. If $t(\alpha)$ and $T(\alpha)$ are chosen such that $\lim _{\alpha \rightarrow \infty} t T=q_{0}$, then, in the notation of Theorem 3.1, $q_{1}=q_{2}=q_{0}$ and (3.12) becomes (6.12) with the constant in (6.11). If however $t(\alpha)$ and $T(\alpha)$ are chosen such that $\lim \inf _{\alpha \rightarrow \infty} t T<q_{0}$ or $\lim \sup _{\alpha \rightarrow \infty} t T>q_{0}$ or both, then Theorems 3.1 and 5.1 imply existence of functions $u(x)$ in the required class for which

$$
\underset{\alpha \rightarrow \infty}{\limsup }|F(t)-S(T)|>A_{0} \limsup _{x \rightarrow \infty}|x u(x)|
$$

when $A_{0}$ is the constant in (6.11).

Theorem 6.1 and a little further argument provide proofs of the following two theorems.

Theorem 6.2. The constant $A_{0}$ in (6.11) is the least constant $B_{1}$ with the following property. If $u(x)$ is integrable over each finite interval and if lim sup $|x u(x)|<\infty$, then it is possible to choose a positive function $t(T)$ independent of $u(x)$ such that $\lim _{T \rightarrow \infty} t(T)=0$ and

$$
\limsup _{T \rightarrow \infty}|F(t(T))-S(T)| \leqq B_{1} \limsup _{x \rightarrow \infty}|x u(x)| .
$$

Theorem 6.3. The constant $A_{0}$ in (6.11) is the least constant $B_{2}$ with the following property. If $u(x)$ is integrable over each finite interval and if lim sup $|x u(x)|<\infty$, then it is possible to choose a positive function $T(t)$ independent of $u(x)$ such that $\lim _{t \rightarrow 0} T(t)=\infty$ and

$$
\limsup _{t \rightarrow 0}|F(t)-S(T(t))| \leqq B_{2} \limsup _{x \rightarrow \infty}|x u(x)|
$$

We have stated Theorems 6.2 and 6.3, which cannot be improved by methods heretofore used in this paper, in the above forms in order to direct attention to the questions whether we can reduce the constants $B_{1}$ and $B_{2}$ if we remove the restrictions that the functions $T(t)$ and $t(T)$ be independent of $u(x)$. These questions appear to be difficult. The author knows of no case in which the problem relating to $B_{2}$ has been solved, either for kernel transformations of functions or for matrix or function transformations of series and sequences. The problem relating to $B_{1}$ was solved for the Abel power series transformation of series by Agnew [1945]; see also Agnew [1949].

In the following sections we shall assume that $\phi(x)$ satisfies all of the conditions we have imposed upon it and the additional condition $\phi(x) \geqq 0$, and shall show that $B_{1}$ cannot be reduced even when $u(x)$ is further restricted to the class of bounded real continuous functions for which $S(T)$ is bounded. Thus, for example, we shall solve the problem involving $B_{1}$ for the transformations of Laplace, Riesz, Stieltjes, and Lambert; but the Riemann transformations for which $\phi(x)=[(\sin x) / x]^{r}$ with $r$ an odd positive integer escape our treatment. All problems relating to $B_{2}$ remain unsolved. 
7. The transform of a simple special function. Let $\phi(x)$ satisfy, in addition to the conditions imposed in $\$ 1$, the condition

$$
\phi(x) \geqq 0,
$$$$
x>0 \text {. }
$$

As a prelude to the construction of an example from which conclusions can be drawn, we derive the principal property, expressed in (7.4), of the transform of a special function $u_{n}(x)$. The example is analogous to the example of Agnew $[1945$, p. 31$]$ which involves Abel power series transforms of series. In spite of its more special character, the example of Agnew [1945] is more abstruse than the present one, partly because series are in this case more awkward than functions and partly because formulas and methods analogous to those used earlier in this paper did not exist in 1945 and best values had to be discovered in the process of construction of the example.

Let $\lambda$ and $x_{n}$ be positive numbers subject to determination later, and let $u_{n}(x)$ be the function defined by

$$
\begin{array}{rlr}
x u_{n}(x) & =0, & x<x_{n}, \\
& =1, & x_{n} \leqq x<\lambda x_{n}, \\
& =-1, & \lambda x_{n} \leqq x<\lambda \lambda x_{n}, \\
& =0, & \lambda \lambda x_{n} \leqq x .
\end{array}
$$

The function $S_{n}(t)$ defined by

$$
S_{n}(T)=\int_{0}^{T} u_{n}(x) d x
$$

is 0 when $T<x_{n}$, is $\log T-\log x_{n}$ when $x_{n} \leqq T<\lambda x_{n}$, is $2 \log \lambda+\log x_{n}-\log T$ when $\lambda x_{n} \leqq T<\lambda \lambda x_{n}$, and is 0 when $\lambda \lambda x_{n} \leqq T$. Thus $S_{n}(t)$ is nonnegative and has the maximum value $\log \lambda$. The transform $F_{n}(t)$ of this function $u_{n}(x)$ is

$$
\begin{aligned}
F_{n}(t) & =\int_{0}^{\infty} \phi(x t) u_{n}(x) d x=\int_{x_{n}}^{\lambda x_{n}} \frac{\phi(x t)}{x} d x-\int_{\lambda x_{n}}^{\lambda \lambda x_{n}} \frac{\phi(x t)}{x} d x \\
& =\int_{x_{n} t}^{\lambda x_{n} t} \frac{\phi(x)}{x} d x-\int_{\lambda x_{n} t}^{\lambda \lambda x_{n} t} \frac{\phi(x t)}{x} d x \\
& =\log \lambda-\int_{x_{n} t}^{\lambda x_{n} t} \frac{1-\phi(x)}{x} d x-\int_{\lambda x_{n} t}^{\lambda \lambda x_{n} t} \frac{\phi(x)}{x} d x .
\end{aligned}
$$

Let $\epsilon_{1}$ be a fixed positive number. The next and most tedious step in our argument is to show that we can choose a large positive number $\lambda$ such that

$$
F_{n}(t)<\epsilon_{1}+\log \lambda-A_{0},
$$$$
t>0,
$$

where $A_{0}$ is the constant in (6.11). To obtain (7.4) from (7.3) we need, for each $t>0$, the inequality 


$$
\begin{aligned}
\int_{x_{n} t}^{\lambda x_{n} t} \frac{1-\phi(x)}{x} d x & +\int_{\lambda x_{n} t}^{\lambda \lambda x_{n} t} \frac{\phi(x)}{x} d x \\
& >-\epsilon_{1}+\int_{0}^{q 0} \frac{1-\phi(x)}{x} d x+\int_{q_{0}}^{\infty} \frac{\phi(x)}{x} d x .
\end{aligned}
$$

To simplify writing, we denote the sum of the two integrals on the left by $L$ (left integrals) and denote the sum of the two integrals on the right by $R$ (right integrals). Choose $h_{1}$ and $h_{2}$ such that $0<h_{1}<q_{0}<h_{2}<\infty$ and

$$
\begin{aligned}
& \int_{0}^{h_{1}} \frac{1-\phi(x)}{x} d x<\frac{\epsilon_{1}}{2}, \quad \int_{h_{1}}^{q_{0}} \frac{\phi(x)}{x} d x>R, \\
& \int_{h_{2}}^{\infty} \frac{\phi(x)}{x} d x<\frac{\epsilon_{1}}{2}, \quad \int_{q_{0}}^{h_{2}} \frac{1-\phi(x)}{x} d x>R .
\end{aligned}
$$

Choose $\lambda$ such that $\lambda>h_{2} / h_{1}, \log \lambda>2 R$. With this value of $\lambda$, we prove (7.5).

In case $\lambda \lambda x_{n} t<h_{2}$, we have $\lambda x_{n} t<h_{2} / \lambda<h_{1}$. In this case the first integral in $L$ is small and we use the inequality

$$
L \geqq \int_{\lambda x_{n} t}^{\lambda \lambda x_{n} t} \frac{\phi(x)}{x} d x
$$

If $\lambda \lambda x_{n} t \geqq q_{0}$, then we use (7.71) to obtain

$$
L \geqq \int_{h_{1}}^{q_{0}} \frac{\phi(x)}{x} d x>R .
$$

If $\lambda \lambda x_{n} t<q_{0}$, then $\phi(x)>1 / 2$ over $\lambda x_{n} t \leqq x \leqq \lambda \lambda x_{n} t$ and we use (7.71) to obtain

$$
L \geqq \frac{1}{2} \int_{\lambda x_{n} t}^{\lambda \lambda x_{n} t} \frac{1}{x} d x=\frac{1}{2} \log \lambda>R .
$$

In case $x_{n} t>h_{1}$, we have $\lambda x_{n} t>\lambda h_{1}>h_{2}$. In this case the second integral in $L$ is small and we use the inequality

$$
L \geqq \int_{x_{n} t}^{\lambda x_{n} t} \frac{1-\phi(x)}{x} d x .
$$

If $x_{n} t \leqq q_{0}$, then we use (7.81) to obtain

$$
L \geqq \int_{q_{0}}^{h_{2}} \frac{1-\phi(x)}{x} d x>R .
$$

If $q_{0}<x_{n} t$, then $\phi(x)<1 / 2$ over $x_{n} t<x<\lambda x_{n} t$ and we use (7.81) to obtain

$$
L \geqq \frac{1}{2} \int_{x_{n} t}^{\lambda x_{n} t} \frac{1}{x} d x=\frac{1}{2} \log \lambda>R .
$$


In the remaining case in which $x_{n} t<h_{1}<q_{0}<h_{2} \leqq \lambda \lambda x_{n} t$ we start with

$$
L=\int_{x_{n} t}^{\lambda x_{n} t} \frac{1-\phi(x)}{x} d x+\int_{\lambda x_{n} t}^{\lambda \lambda x_{n} t} \frac{\phi(x)}{x} d x
$$

and observe that, since $\phi(x)>1 / 2$ when $x<q_{0}$ and $\phi(x)<1 / 2$ when $x>q_{0}$,

$$
L \geqq \int_{x_{n} t}^{q_{0}} \frac{1-\phi(x)}{x} d x+\int_{q_{0}}^{\lambda \lambda x_{n} t} \frac{\phi(x)}{x} d x .
$$

With the aid of (7.61) and (7.62), we now obtain

$$
L \geqq \int_{h_{1}}^{q_{0}} \frac{1-\phi(x)}{x} d x+\int_{q_{0}}^{h_{2}} \frac{\phi(x)}{x} d x>-\epsilon_{1}+R .
$$

This completes the proof of (7.5) and hence also (7.4).

8. The transform of a special function. We now prove the following theorem.

THEOREM 8.1. Let $\phi(x)$ satisfy, in addition to our original hypotheses, the condition $\phi(x) \geqq 0$. Let $\epsilon>0$ and let $A_{0}$ be the constant in (6.11). Then there is $a$ positive constant $\lambda>1$ and $a$ function $u(x)$, real and continuous over $x \geqq 0$, such that

$$
\begin{aligned}
-1 \leqq x u(x) \leqq 1, & x \geqq 0, \\
0 \leqq \int_{0}^{T} u(x) d x \leqq \log \lambda, & T \geqq 0, \\
\limsup _{T \rightarrow \infty} \int_{0}^{T} u(x) d x=\log \lambda, &
\end{aligned}
$$

and

$$
0<\int_{0}^{\infty} \phi(x t) u(x) d x<\epsilon+\log \lambda-A_{0},
$$

With $\epsilon>0$ given in Theorem 8.1 , let $\epsilon_{1}=\epsilon / 4$ and $\lambda$ be fixed as in $\$ 7$. We use the notation and functions of $\$ 7$. Let $x_{1}>1$ so that $u_{1}(x), S_{1}(T)$, and $F_{1}(t)$ are determined. Since $F_{1}(t) \rightarrow 0$ as $t \rightarrow 0$, we can choose $t_{1}>0$ such that

$$
\left|F_{1}(t)\right|<\epsilon_{1},
$$$$
0<t<t_{1} \text {. }
$$

Then choose $x_{2}$ such that $x_{2}>\lambda \lambda x_{1}$ and

$$
\int_{x_{2}}^{\infty} \frac{\phi(x t)}{x} d x<\epsilon_{1}, \quad t \geqq t_{1} ;
$$

in order to obtain (8.3), it is sufficient to choose $x_{2}$ so great that 


$$
\int_{t_{1} x_{2}}^{\infty} \frac{\phi(x)}{x} d x<\epsilon_{1}
$$

and this choice of $x_{2}$ is possible because of (1.2). We now choose $t_{2}$ such that the formulas $0<t_{n-1}<t_{n-2}$ and

$$
\sum_{k=1}^{n-1}\left|F_{k}(t)\right|<\epsilon_{1}, \quad 0<t \leqq t_{n-1},
$$

hold when $n=3$. Choose $x_{3}$ such that the formulas $x_{n+1}>\lambda \lambda x_{n}$ and

$$
\int_{x_{n+1}}^{\infty} \frac{\phi(x t)}{x} d x<\epsilon_{1}, \quad t \geqq t_{n},
$$

hold when $n=2$. Continue to choose, in the order given, numbers $t_{3}, x_{4}$, $t_{4}, x_{5}, \cdots$ such that $t_{n} \rightarrow 0,0<t_{n+1}<t_{n}, x_{n} \rightarrow \infty, x_{n+1}>\lambda \lambda x_{n}$, and the formulas (8.4) and (8.5) hold.

Let $U(x)$ be the function defined by

$$
U(x)=\sum_{k=1}^{\infty} u_{k}(x)
$$$$
x>0 \text {. }
$$

Since the functions $u_{k}(x)$ differ from zero only over distinct intervals $x_{k}$ $\leqq x \leqq \lambda \lambda x_{k}$, properties of the functions $u_{k}(x)$ imply that $U(x)$ has the properties required of $u(x)$ in (8.11), (8.12), and (8.13). The transform $F(t)$ of $U(x)$ is, like the functions $F_{k}(t)$, positive. We find that

$$
\begin{aligned}
F(t)= & \int_{0}^{\infty} \phi(x t) U(x) d x \\
= & \sum_{k=1}^{n-1} \int_{x_{k}}^{\lambda \lambda x_{k}} \phi(x t) U(x) d x \\
& +\int_{x_{n}}^{\lambda \lambda x_{n}} \phi(x t) U(x) d x+\int_{x_{n+1}}^{\infty} \phi(x t) U(x) d x .
\end{aligned}
$$

When $t_{n} \leqq t \leqq t_{n-1}$,

$$
F(t)<\sum_{k=1}^{n-1} F_{k}(t)+F_{n}(t)+\int_{x_{n+1}}^{\infty} \frac{\phi(x t)}{x} d x
$$

so that (8.4) and (8.5) give

$$
F(t)<2 \epsilon_{1}+F_{n}(t)
$$

and (7.4) then gives

$$
F(t)<3 \epsilon_{1}+\log \lambda-A_{0} .
$$


Thus, since $3 \epsilon_{1}<4 \epsilon_{1}=\epsilon$, the function $U(x)$ has all of the properties of $u(x)$ required by Theorem 8.1 except that $U(x)$ is not continuous.

It is a simple matter to define a continuous function $u(x)$ such that

$$
\int_{0}^{\infty}|u(x)-U(x)| d x<\epsilon_{1}
$$

and $u(x)$ has all of the essential properties of $U(x)$ that we have used. For example, we could make $u(x)$ linear over short intervals $x_{n}-p_{n} \leqq x_{n} \leqq x_{n}$ and $\lambda x_{n}-q_{n} \leqq x \leqq \lambda x_{n}$ in such a way that $u\left(x_{n}-p_{n}\right)=u\left(\lambda x_{n}\right)=0, u(x)=U(x)$ when $x_{n} \leqq x \leqq \lambda x_{n}-q_{n}$, and

$$
\int_{x_{n}-p_{n}}^{\lambda x_{n}} u(x) d x=\int_{x_{n}-p_{n}}^{\lambda x_{n}} U(x) d x .
$$

Applying the same process with small intervals $\lambda x_{n} \leqq x \leqq \lambda x_{n}+r_{n}$ and $\lambda \lambda x_{n}$ $\leqq x \leqq \lambda \lambda x_{n}+s_{n}$ in such a way that

$$
\int_{x_{n}-p_{n}}^{\lambda \lambda x_{n}+s_{n}}|u(x)-U(x)| d x<\frac{\epsilon_{1}}{2^{n+1}},
$$

we obtain a function $u(x)$ of the type desired. The transform of $u(x)$, which will be positive, is such that

$$
\begin{aligned}
\int_{0}^{\infty} \phi(x t) u(x) d x< & \int_{0}^{\infty} \phi(x t) U(x) d x \\
& +\int_{0}^{\infty} \phi(x t)|u(x)-U(x)| d x \\
< & 3 \epsilon_{1}+\log \lambda-A_{0}+\epsilon_{1}=\epsilon+\log \lambda-A_{0} .
\end{aligned}
$$

In estimating the integrals, we use (8.64), (8.7), and the fact that $0 \leqq \phi(x) \leqq 1$. This completes the proof of Theorem 8.1.

9. The constant in Theorem 6.2. For comparison with Theorem 6.2, and to invite further study of Theorem 6.3, we state the following theorem.

THEOREM 9.1. Let $\phi(x)$ satisfy, in addition to our original hypotheses, the condition $\phi(x) \geqq 0$. Then the constant $A_{0}$ in (6.11) is the least constant $B_{1}$ with the following property. If $u(x)$ is a real continuous function for which $\lim \sup |x u(x)|<\infty$ and $S(T)$ is bounded, then it is possible to choose a positive function $t(T)$, which may depend upon $u(x)$, such that

$$
\limsup _{T \rightarrow \infty}|F(t(T))-S(T)| \leqq B_{1} \limsup _{x \rightarrow \infty}|x u(x)| .
$$

That the constant $A_{0}$ will serve for each function $u(x)$ in the more restricted class of Theorem 9.1 follows from the fact that it serves for the larger class in Theorem 6.2. That no constant $B_{1}$ less than $A_{0}$ can serve is implied 
by Theorem 8.1. In fact the three relations (8.11), (8.13), and (8.14) imply that, for the special function $u(x)$ of Theorem 8.1 , we have

$$
\limsup _{T \rightarrow \infty}[S(T)-F(t(T))] \geqq\left[A_{0}-\epsilon\right] \limsup _{x \rightarrow \infty}|x u(x)|
$$

for every function $t(T)$.

10. Conclusion. As was indicated in $\S 1$, the significance of Theorems 3.1, $5.1,6.1,6.2,6.3$, and 9.1 lies in the fact that they give information about $|F(t)-S(T)|$ when neither $\lim _{t \rightarrow 0} F(t)$ nor $\lim _{T \rightarrow \infty} F(t)$ is assumed to exist. We nevertheless examine implications of the theorems in cases where one of the latter limits exists.

In case $u(x)$ is integrable over each finite interval, lim sup $|x u(x)|<\infty$, and $\lim _{T \rightarrow \infty} S(T)=S$, Theorem 6.3 implies that

$$
\limsup _{t \rightarrow 0}|F(t)-S| \leqq A_{0} \limsup _{x \rightarrow \infty}|x u(x)|
$$

where $A_{0}$ is the constant in (2.2) and (6.11). If the transformation (1.5) is known to be regular, in which case existence of $S=\lim S(T)$ implies $\lim F(t)$ $=S$ even without the Tauberian condition $\lim \sup |x u(x)|<\infty$, then (10.1) is completely without interest. If however the transformation (1.5) is not regular (as is true for the classic Riemann transformation $R_{1}$ for which $\left.\phi(x)=x^{-1} \sin x\right)$, then (10.1) is significant. In particular, (10.1) implies that the transformation is regular over the class of functions $u(x)$ which are integrable over each finite interval and satisfy the condition $\lim x u(x)=0$. One could presume that it has previously been discovered that the Riemann transformation $R_{\mathbf{1}}$ has this property, but the author cannot give a reference.

In case $u(x)$ is integrable over each finite interval, lim sup $|x u(x)|<\infty$, and $\lim _{t \rightarrow 0} F(t)=F$, Theorem 6.2 implies that

$$
\limsup _{T \rightarrow \infty}|F-S(T)| \leqq A_{0} \limsup _{x \rightarrow \infty}|x u(x)|
$$

where $A_{0}$ is the constant in (2.2) and (6.11). If the transformation (1.5) is one for which we know an " $O$ Tauberian theorem" to the effect that existence of $F=\lim F(t)$ and the Tauberian condition $\lim \sup |x u(x)|<\infty$ imply $\lim S(T)$ $=F$, then (10.2) is completely without interest. If no such " $O$ Tauberian theorem" is available, then it is of interest to note that (10.2) implies an "o Tauberian theorem" to the effect that existence of $F=\lim F(t)$ and the Tauberian condition $\lim |x u(x)|=0$ imply that $\lim S(T)=F$.

Added April 28, 1952. Garten [1951] has recently determined the best function $A_{r}^{*}(q)$ such that

$$
\lim \sup \left|c_{p}^{(r)}-s_{n}\right| \leqq A_{r}^{*}(q) \limsup _{m \rightarrow \infty}\left|m u_{m}\right|
$$

whenever $\sum u_{n}$ is a series with partial sums $s_{n}$ and Cesàro transform $c_{p}^{(r)}$ of 
positive integer order $r$, and the indices in the left member become infinite with $p=[n / q]$ or $n=[q p]$. As could be expected, the Garten results involving Cesàro transforms of series are, insofar as they are comparable, very similar to the results we have obtained for Riesz transforms of functions. For Cesàro orders 1, 2, and 3, numerical constants are given by Garten $[1951$, p. 323] to 5 or 6 decimal places; these agree with the values given between $(2.52)$ and (2.53) above.

\section{REFERENCES}

R. P. Agnew, [1939] Properties of generalized definitions of limit, Bull. Amer. Math. Soc. vol. 45, pp. 689-730.

- [1945] Abel transforms of Tauberian series, Duke Math. J. vol. 12, pp. 27-36. vol. 50, pp. $110-117$.

Hubert Delange, [1950] Sur les théorèmes inverses des procédés de sommation. I, Ann. École Norm. (3) vol. 67 , pp. 99-160.

Viktor Garten, [1951] Über Taubersche Konstanten bei Cesdroschen Mittelbildungen, Comment. Math. Helv. vol 25, pp. 311-335.

Hugo Hadwiger, [1944] Über ein Distanztheorem bei der A-Limitierung, Comment. Math. Helv. vol. 16, pp. 209-214.

G. H. Hardy, [1949] Divergent series, Oxford, $396 \mathrm{pp.}$

C. T. Rajagopal, [1950] On a generalization of Tauber's theorem, Comment. Math. Helv. vol. 24, pp. 219-231.

CORNELl University, ITHACA, $\mathrm{N}$. Y. 